\title{
Gene of the month: TLE 1
}

\author{
Karen Pinto, ${ }^{1}$ Runjan Chetty (1) ${ }^{2}$
}

${ }^{1}$ Pathology, Kuwait Cancer Control Center, Shuwaikh, Al Asimah, Kuwait

${ }^{2}$ Department of Histopathology, Brighton and Sussex University Hospitals NHS Trust, Brighton, UK

\section{Correspondence to}

Professor Runjan Chetty, Department of Histopathology, Brighton and Sussex University Hospitals NHS Trust, Brighton BN2 5BE, UK; runjan.chetty@ gmail.com

Accepted 5 December 2020 Published Online First 13 January 2021

\section{Check for updates}

(c) Author(s) (or their employer(s)) 2021. No commercial re-use. See rights and permissions. Published by BMJ.

To cite: Pinto K Chetty R. J Clin Pathol 2021:74:137-140.

\section{ABSTRACT}

TLE 1 is the human homologue belonging to a family of four genes and is located on chromosome 9q21. It consists of 19 exons. Although it does not bind directly to DNA, it acts as a repressor of several signalling pathways via transcription factors. TLE1 protein has several physiological roles in embryogenesis, haematopoiesis, general differentiation, and both neuronal and eye development. Much attention was focused on its expression in the tumour cell nuclei of synovial sarcoma (SS). However, several other soft tissue tumours that do and do not share morphological similarity with SS also display nuclear immunoreactivity for TLE 1 ; hence, caution in interpretation is advocated.

\section{GENE STRUCTURE AND PROTEIN}

The Transducin-like enhancer of split 1 (TLE 1) gene belongs to a family of four genes, is located on chromosome $9 \mathrm{q} 21$ and is consists of 19 exons. TLE1 protein is the human homologue of the Drosophila groucho protein. It encodes a non-DNA binding, 770 amino acid transcriptional protein that serves as a co-repressor of other transcription factors and signalling pathways. TLE1 protein forms homo-oligomers and hetero-oligomers with several proteins, and after binding, it acts to inhibit transcriptional activity, especially in the Wnt signalling pathway where it interacts with $\beta$-catenin and T-cell factor.

\section{PHYSIOLOGICAL ROLE}

TLE1 protein plays a normal, physiological role in several processes including during embryogenesis, haematopoiesis, epithelial differentiation, segmentation and neuronal/eye development (see figure 1).

\section{TLE 1 IN DISEASE Non-cancer}

See figure 2.

\section{Inflammation}

The nuclear factor-kappa B (NF- $\kappa \mathrm{B})$ signalling pathway controls the immune response; hence, it plays a role in inflammation. TLE 1 represses NF- $\mathrm{KB}$ activity. Thus, the loss of TLE1 results in excessive activation of NF- $\mathrm{KB}$-mediated inflammatory pathways associated with increased expression of inflammatory cytokines and chemokines in the skin, lung and intestine. ${ }^{1}$ Interactions of TLE1 and nucleotide-binding oligomerisation domaincontaining protein 2 (NOD2) are involved with inflammatory bowel disease. NOD2 gene was the first susceptibility gene identified in Crohn's disease (CD). Nimmo et $a l^{2}$ suggested that TLE1 can regulate NOD2 functions. They realised single nucleotide polymorphisms (SNP) in TLE1 (intronic SNP rs6559629) result in a TLE risk allele which unmasks the mutant NOD2 resulting in CD (ileal phenotype).

Diabetes mellitus (type 2)

TLE1 is a regulator of pancreatic islet $\beta$-cell identity. Armour et $a l^{3}$ indicated that reduced expression of TLE1 in islet cells is inversely correlated with $\alpha / \beta$ cell ratio. Decreased TLE1 causes an increase in glucagon gene mRNA and misexpression of glucagon in islet cells, which leads to a loss of cellular identity potentially leading to $\beta$-cell to $\alpha$-cell conversion.

\section{Postnatal microcephaly}

In postnatal microcephaly, although the child has a normal head size at birth, there is a subsequent deceleration of the head circumference caused by mutations in genes responsible for the development of the forebrain. Mutations in genes like CASK, CDKL5, CREBBP, EP300, FOXG1 and SLC9A6 are already known, and recently, TLE1 has been added to the list. A homozygous missense mutation in TLE1 results in autosomal recessive postnatal microcephaly. ${ }^{45}$

\section{Sheehan syndrome}

Sheehan syndrome is postpartum hypopituitarism spawned due to necrosis of the pituitary gland as a result of severe hypotension or shock during or after childbirth. Diri et $a l^{6}$ studied patients with Sheehan syndrome and concluded that they have abnormal expressions of TLE1 (and TLE3). They suggested this may disrupt differentiation of pituitary cells and lead to a genetic predisposition to Sheehan syndrome.

\section{Role in cancer}

See figure 3.

Even though the TLE1 protein does not bind to DNA directly, it attaches to specific regions of DNA via its association with a variety of transcription factors. For example, TLE1 binds to the histone, $\mathrm{H} 3$, with resultant modification of chromatin structure, thereby allowing TLE1 to regulate gene expression.

TLE1 exerts its influence in three ways: (1) by downregulating transcriptional activators, (2) by enhancing transcriptional repressors and (3) by conversion of transcriptional activators into repressors.

TLE1 binds to basic helix loop helix (bHLH) proteins and represses target genes in three main pathways: 


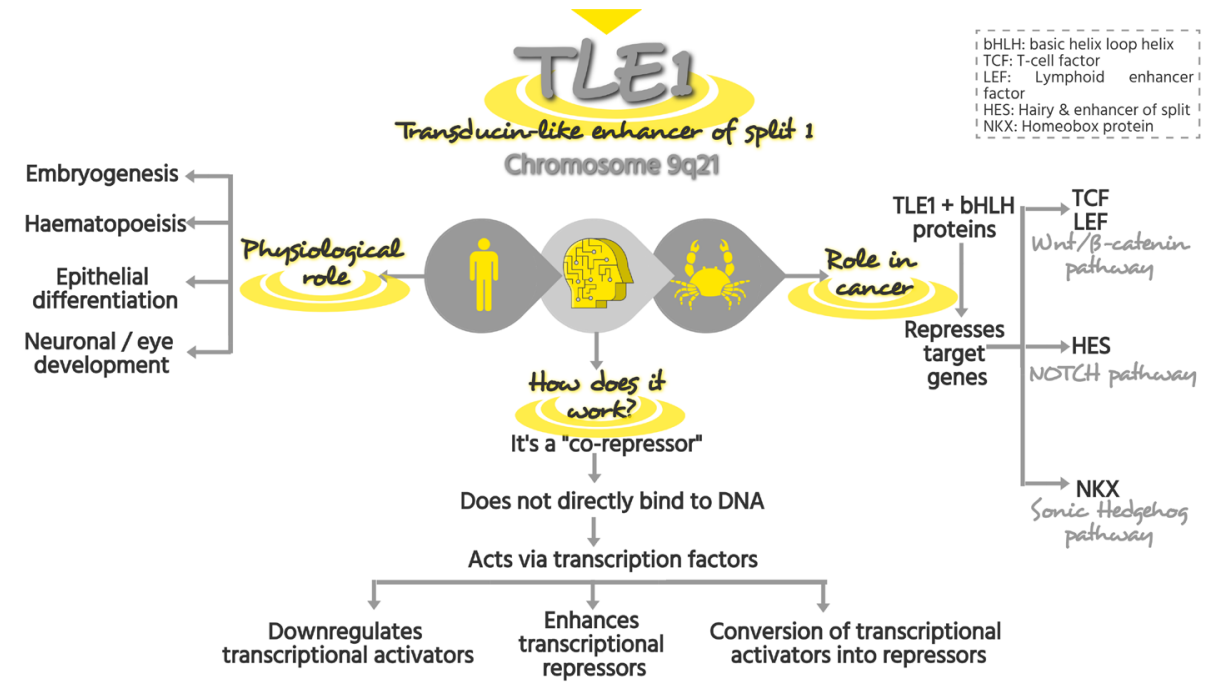

Figure 1 Schematic infographic illustrating the mode of action of the TLE1 gene.

- TCF/LEF (T-cell factor/Lymphoid enhancer factor) in the $\mathrm{WNT} / \beta$-catenin pathway.

- HES (hairy and enhancer of split) in the NOTCH pathway.

- NKX (homeobox protein) in the sonic Hedgehog pathway.

A dysregulation in any of these can act as a driver in tumourigenesis. $^{7}$

\section{Synovial sarcoma}

The SS18-SSX gene fusion is specific to synovial sarcoma (SS) and is its primary genetic driver mutation. Su et $a l^{89}$ determined that SS18-SSX forms a functional endogenous complex with activating transcription factor 2 (ATF2) and TLE1 which represses ATF2 target genes and resulted in abnormal transcriptional activities which drive the malignant transformation in SS. SS18-SSX acts as a frame to link these proteins together (although independently through different protein domains) resulting in an SS18-SSX/TLE1/ATF2 complex which directly binds to the EGR1 (early growth response 1) promoter. In healthy cells, EGR1 impairs tumour development and induces expression of tumour suppressors like $p 53, T G F-\beta, P T E N$ and so on. However, when SS18-SSX/TLE1/ATF2 complex binds to EGR1, it represses its transcription and boosts oncogenesis.
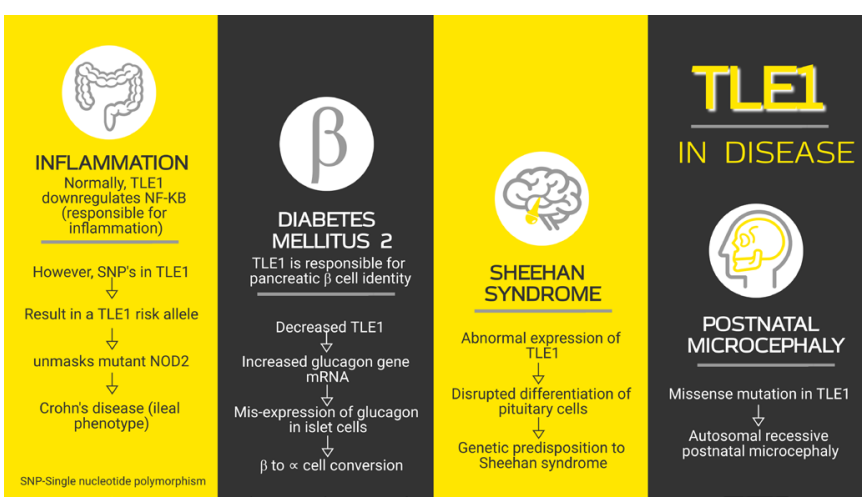

Figure 2 An infographic elucidating the role of TLE1 in disease. NF$\kappa \mathrm{B}$, nuclear factor-kappa $\mathrm{B}$.

\section{Diagnostic application of TLE1 immunohistochemistry}

From a practical point of view, antibodies have been developed to TLE1 and its immunohistochemical application in soft tissue sarcoma pathology which has been useful in detecting SSs (figure 4). TLE immunohistochemistry is by no means specific for $S$ as several other soft tissue lesions have been noted to be positive for TLE1 antibody: neurofibroma, schwannoma, malignant peripheral nerve sheath tumour, solitary fibrous tumour, rhabdomyosarcoma, leiomyosarcoma, Ewing sarcoma family, high-grade chondrosarcoma and clear cell sarcoma of soft parts.

Thus, judicious application and interpretation of the antibody in soft tissue tumours is advocated. In other words, a spindle cell soft tissue tumour that is TLE1 positive is not automatically an SS.

T-cell acute lymphoblastic leukaemia

Riz et $a l^{10}$ studied the role of TLE1 in T-cell acute lymphoblastic leukaemia (T-ALL) with regard to the TLX1 (T-cell leukaemia homeobox 1) gene. TLX1 is a transcription regulator which depending on the availability of transcription cofactors can

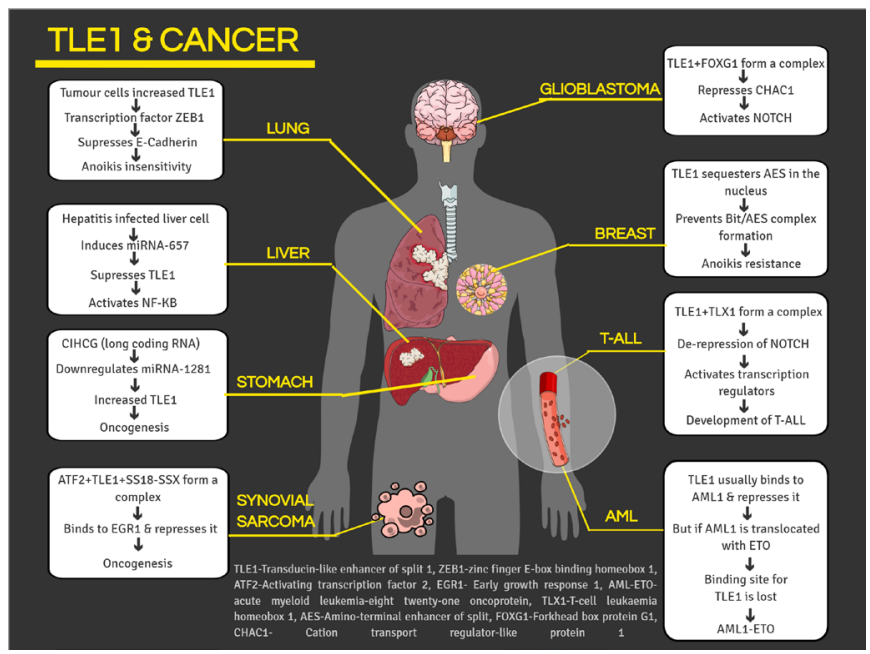

Figure 3 An infographic elucidating the role and mechanism of action of the TLE1 gene in oncogenesis. NF- $\kappa B$, nuclear factor-kappa B. 


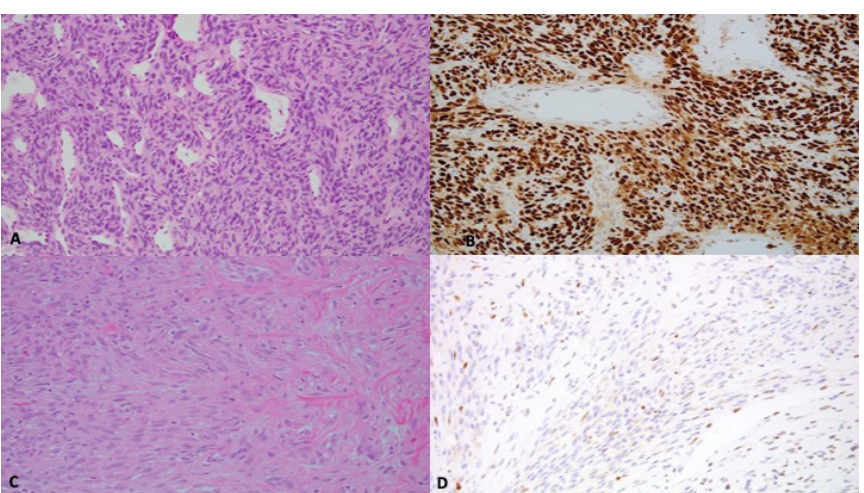

Figure 4 Image featuring TLE1 immunohistochemistry (nuclear stain) in synovial sarcoma.

activate or repress gene expression. TLE1 co-represses NOTCH signalling by establishing negative loops of regulation and mediating NOTCH repressor activity. However, TLX1-TLE1 interaction causes de-repression of NOTCH-induced genes and activation of downstream transcription regulators. They identified TLX1/TLE1/NOTCH network as the cause for developmental arrest and survival of T-ALL. Low expression of the TLE1 immunostain also indicates a poor prognosis in ALL. ${ }^{11}$

\section{Acute myeloid leukaemia}

TLE1 plays a role in the differentiation of immature haematopoietic cells. At the end of haematopoiesis, TLE1 causes the repression of target genes from the AML1, Wnt and $\mathrm{NOTCH}$ signalling pathways permitting committed progenitors to begin to acquire the characteristic differentiation status of mature haematopoietic cells. TLE1 binds to AML1 of the AML/CBF $\alpha$ runt domain transcription factor family. However, this binding site is lost when AML1 is translocated with ETO (Eight-Twenty one oncoprotein) resulting in an imbalance in the signalling pathway, resulting in the development of AML with AML1-ETO fusion. Fraga et al ${ }^{12}$ found that TLE1 undergoes promoter CpG island hypermethylation-associated inactivation in haematologic malignancies, such as diffuse large B-cell lymphoma and AML by disrupting cell differentiation and activation growth-suppression pathways.

\section{Glioblastoma}

Forkhead box protein G1 (FOXG1) is a transcription factor that promotes the proliferation of progenitor cells in the cerebral cortex. FOXG1 forms complexes with TLE1 which then repress genes (Cation transport regulator-like protein 1-CHAC1) that negatively regulate $\mathrm{NOTCH}$ signalling in brain-tumour initiating cells). CHAC1 acts as a pro-apoptotic factor and is the target for FOXG1:TLE1 transcription repression complexes which then promotes glioma cell survival. ${ }^{13}$

\section{Lung cancer}

In healthy cells, anoikis is a form of programmed cell death that occurs in anchorage-dependent cells when they detach from the surrounding extracellular matrix. Tumour cells have the unique ability to evade this process, grow independently and metastasise. Yao et $a l^{14}$ studied the role of TLE1 and its interaction with E-cadherin which is a key regulator of epithelial-mesenchymal transition in lung cancer cells. When a healthy cell loses its attachment, E-cadherin expression is transcriptionally induced which then stimulates anoikis. However, in malignant bronchial epithelial cells, there is an increased expression of TLE1 which causes suppression of E-cadherin via transcription factor Zinc finger E-box binding homeobox 1 (ZEB1) which confers enhanced anoikis insensitivity and anchorage-independent growth to the malignant cells.

\section{Breast cancer}

Because TLE1 is a 'co-repressor', it does not bind to DNA directly but binds to other DNA-binding transcription factors to form multiprotein complexes. Just like in the lung, TLE1 plays a role in breast cancer cell anoikis resistance but through the Bit1 anoikis pathway. Mitochondrial Bit forms a complex with AES (amino-terminal enhancer of split) in the cytoplasm leading to apoptosis. However, in breast cancer cells where TLE1 is overexpressed, TLE1 sequesters AES in the nucleus, thus preventing the formation of Bit1-AES complexes. ${ }^{15}$

\section{Gastric cancer}

Long coding RNAs (LncRNA) are a subtype of RNAs that are incapable of protein coding. However, they can bind to proteins, chromosomes and other macromolecules involved in the regulatory network of endogenous RNA which play a role in tumourigenesis by competitively binding to microRNA (miRNA). In gastric cancer, GIHCG is the LncRNA that downregulates miR-1281, a miRNA. TLE1 is the target gene for miR-1281, and they have an inverse relationship. So the downregulation of miR-1281 causes an increased TLE1 expression in malignant gastric epithelial cells which results in cell proliferation and migration. ${ }^{16}$ TLE1 immunostain expression in gastric cancers is associated with male gender, less frequent lymphatic and perineural invasion, intestinal subtype (Lauren classification), good histologic grade, early pathologic T-stage and longer disease-free survival. $^{17}$

\section{Hepatocellular cancer}

Hepatitis viral proteins alter host cell miRNA expression. In hepatocellular cancer (HCC), miR-657 plays a pivotal role. In infected cells, viral proteins induce miRNA-657 which directly suppresses TLE1. As normal hepatic cells progress from dysplasia to cancer, their TLE1 mRNA gradually decreases. As outlined previously, TLE1 and NF- $\kappa \mathrm{B}$ pathways are related. In the liver, NF- $\kappa \mathrm{B}$ modulates basic cellular processes like hepatic apoptosis, proliferation and cancer development. Suppression of TLE1 activates NF- $\kappa \mathrm{B}$ pathways, which contributes to hepatocellular carcinogenesis. $^{18}$

\section{Take home messages}

TLE1 belongs to a family of transcription repressors and is located on chromosome 9q21.

- TLE1 protein binds to proteins in three pathways: Wnt, Notch and sonic Hedgehog pathways.

- TLE1 inhibits transcription activators leading to repression of transcription.

- TLE1 overexpression is characteristic of synovial sarcoma, especially those with the $(X ; 18)$ translocation.

- TLE1 is also expressed immunohistochemically in a variety of other soft tissue tumours and epithelial malignancies.

Handling editor Dhirendra Govender.

Twitter Runjan Chetty @runjanchetty 
Acknowledgements The authors would like to thank Dr Vikram Deshpande, Massachusetts General Hospital and Harvard Medical School, Boston, USA, for the immunohistochemical images of TLE1.

Contributors KP and RC contributed equally to concept, collation of data, design and writing of the manuscript.

Funding The authors have not declared a specific grant for this research from any funding agency in the public, commercial or not-for-profit sectors.

Competing interests RC is a consultant for Deciphex Ltd, Ireland.

Patient consent for publication Not required.

Provenance and peer review Not commissioned; externally peer reviewed.

\section{ORCID iD}

Runjan Chetty http://orcid.org/0000-0002-2124-515X

\section{REFERENCES}

1 Ramasamy S, Saez B, Mukhopadhyay S, et al. Tle1 tumor suppressor negatively regulates inflammation in vivo and modulates NF-KB inflammatory pathway. Proc Natl Acad Sci U S A 2016;113:1871-6.

2 Nimmo ER, Stevens C, Phillips AM, et al. Tle1 modifies the effects of NOD2 in the pathogenesis of Crohn's disease. Gastroenterology 2011;141:972-81.

3 Armour SL, Anderson SJ, Richardson SJ, et al. Reduced expression of the coregulator TLE1 in type 2 diabetes is associated with increased islet $\alpha$-Cell number. Endocrinology 2020;161. doi:10.1210/endocr/bqaa011. [Epub ahead of print: 01 Apr 2020].

4 Cavallin M, Maillard C, Hully M, et al. Tle1, a key player in neurogenesis, a new candidate gene for autosomal recessive postnatal microcephaly. Eur J Med Genet 2018;61:729-32.

5 Seltzer LE, Paciorkowski AR. Genetic disorders associated with postnatal microcephaly. Am J Med Genet C Semin Med Genet 2014;166C:140-55.
6 Diri H, Sener EF, Bayram F, et al. Genetic disorders of pituitary development in patients with Sheehan's syndrome. Acta Endocrinol 2016;12:413-7.

7 El Beaino M, Jupiter DC, Assi T, et al. Diagnostic value of TLE1 in synovial sarcoma: a systematic review and meta-analysis. Sarcoma 2020;2020:1-6.

8 Su L, Sampaio AV, Jones KB, et al. Deconstruction of the SS18-SSX fusion oncoprotein complex: insights into disease etiology and therapeutics. Cancer Cell 2012;21:333-47.

9 Lubieniecka JM, de Bruijn DRH, Su L, et al. Histone deacetylase inhibitors reverse SS18-SSX-mediated polycomb silencing of the tumor suppressor early growth response 1 in synovial sarcoma. Cancer Res 2008;68:4303-10.

10 Riz I, Hawley TS, Luu TV, et al. TLX1 and Notch coregulate transcription in T cell acute lymphoblastic leukemia cells. Mol Cancer 2010;9:181.

11 Brassesco MS, Pezuk JA, Cortez MA, et al. Tle1 as an indicator of adverse prognosis in pediatric acute lymphoblastic leukemia. Leuk Res 2018;74:42-6.

12 Fraga MF, Berdasco M, Ballestar E, et al. Epigenetic inactivation of the Groucho homologue gene TLE1 in hematologic malignancies. Cancer Res 2008;68:4116-22.

13 Dali R, Verginelli F, Pramatarova A, et al. Characterization of a FOXG1:TLE1 transcriptional network in glioblastoma-initiating cells. Mol Oncol 2018;12:775-87.

14 Yao X, Pham T, Temple B, et al. Tle1 inhibits anoikis and promotes tumorigenicity in human lung cancer cells through ZEB1-mediated E-cadherin repression. Oncotarget 2017:8:72235-49.

15 Brunquell C, Biliran $\mathrm{H}$, Jennings $\mathrm{S}$, et al. Tle 1 is an anoikis regulator and is downregulated by Bit1 in breast cancer cells. Mol Cancer Res 2012;10:1482-95.

16 Liu G, Jiang Z, Qiao M, et al. Lnc-GIHCG promotes cell proliferation and migration in gastric cancer through miR-1281 adsorption. Mol Genet Genomic Med 2019;7:e711.

17 Lee J-H, Son M-W, Kim K-J, et al. Prognostic and clinicopathological significance of Transducer-Like enhancer of split 1 expression in gastric cancer. J Gastric Cancer 2016;16:21-7.

18 Zhang L, Yang L, Liu X, et al. MicroRNA-657 promotes tumorigenesis in hepatocellular carcinoma by targeting transducin-like enhancer protein 1 through nuclear factor kappa B pathways. Hepatology 2013;57:1919-30. 